\title{
ON A FIXED POINT THEOREM OF KRASNOSEL'SKII TYPE AND APPLICATION TO INTEGRAL EQUATIONS
}

\author{
LE THI PHUONG NGOC AND NGUYEN THANH LONG
}

Received 15 April 2006; Revised 30 June 2006; Accepted 13 August 2006

This paper presents a remark on a fixed point theorem of Krasnosel'skii type. This result is applied to prove the existence of asymptotically stable solutions of nonlinear integral equations.

Copyright (c) 2006 L. T. P. Ngoc and N. T. Long. This is an open access article distributed under the Creative Commons Attribution License, which permits unrestricted use, distribution, and reproduction in any medium, provided the original work is properly cited.

\section{Introduction}

It is well known that the fixed point theorem of Krasnosel'skii states as follows.

Theorem 1.1 (Krasnosel'skii [8] and Zeidler [9]). Let $M$ be a nonempty bounded closed convex subset of a Banach space $(X,\|\cdot\|)$. Suppose that $U: M \rightarrow X$ is a contraction and $C: M \rightarrow X$ is a completely continuous operator such that

$$
U(x)+C(y) \in M, \quad \forall x, y \in M .
$$

Then $U+C$ has a fixed point in $M$.

The theorem of Krasnosel'skii has been extended by many authors, for example, we refer to $[1-4,6,7]$ and references therein.

In this paper, we present a remark on a fixed point theorem of Krasnosel'skii type and applying to the following nonlinear integral equation:

$$
x(t)=q(t)+f(t, x(t))+\int_{0}^{t} V(t, s, x(s)) d s+\int_{0}^{t} G(t, s, x(s)) d s, \quad t \in \mathbb{R}_{+},
$$

where $E$ is a Banach space with norm $|\cdot|, \mathbb{R}_{+}=[0, \infty), q: \mathbb{R}_{+} \rightarrow E ; f: \mathbb{R}_{+} \times E \rightarrow E$; $G, V: \Delta \times E \rightarrow E$ are supposed to be continuous and $\Delta=\left\{(t, s) \in \mathbb{R}_{+} \times \mathbb{R}_{+}, s \leq t\right\}$.

In the case $E=\mathbb{R}^{d}$ and the function $V(t, s, x)$ is linear in the third variable, (1.2) has been studied by Avramescu and Vladimirescu [2]. The authors have proved the existence 
2 On a fixed point theorem and application

of asymptotically stable solutions to an integral equation as follows:

$$
x(t)=q(t)+f(t, x(t))+\int_{0}^{t} V(t, s) x(s) d s+\int_{0}^{t} G(t, s, x(s)) d s, \quad t \in \mathbb{R}_{+},
$$

where $q: \mathbb{R}_{+} \rightarrow \mathbb{R}^{d} ; f: \mathbb{R}_{+} \times \mathbb{R}^{d} \rightarrow \mathbb{R}^{d} ; V: \Delta \rightarrow M_{d}(\mathbb{R}), G: \Delta \times \mathbb{R}^{d} \rightarrow \mathbb{R}^{d}$ are supposed to be continuous, $\Delta=\left\{(t, s) \in \mathbb{R}_{+} \times \mathbb{R}_{+}, s \leq t\right\}$ and $M_{d}(\mathbb{R})$ is the set of all real quadratic $d \times d$ matrices. This was done by using the following fixed point theorem of Krasnosel'skii type.

Theorem $1.2($ see $[1])$. Let $\left(X,|\cdot|_{n}\right)$ be a Fréchet space and let $C, D: X \rightarrow X$ be two operators.

Suppose that the following hypotheses are fulfilled:

(a) $C$ is a compact operator;

(b) $D$ is a contraction operator with respect to a family of seminorms $\|\cdot\|_{n}$ equivalent with the family $|\cdot|_{n}$;

(c) the set

$$
\left\{x \in X, x=\lambda D\left(\frac{x}{\lambda}\right)+\lambda C x, \lambda \in(0,1)\right\}
$$

is bounded.

Then the operator $C+D$ admits fixed points.

In [6], Hoa and Schmitt also established some fixed point theorems of Krasnosel'skii type for operators of the form $U+C$ on a bounded closed convex subset of a locally convex space, where $C$ is completely continuous and $U^{n}$ satisfies contraction-type conditions. Furthermore, applications to integral equations in a Banach space were presented.

On the basis of the ideas and techniques in $[2,6]$, we consider (1.2). The paper consists of five sections. In Section 2, we prove a fixed point theorem of Krasnosel'skii type. Our main results will be presented in Sections 3 and 4 . Here, the existence solution and the asymptotically stable solutions to (1.2) are established. We end Section 4 by illustrated examples for the results obtained when the given conditions hold. Finally, in Section 5, a general case is given. We show the existence solution of the equation in the form

$$
\begin{aligned}
x(t)= & q(t)+f(t, x(t), x(\pi(t)))+\int_{0}^{t} V(t, s, x(s), x(\sigma(s))) d s \\
& +\int_{0}^{t} G(t, s, x(s), x(\chi(s))) d s, \quad t \in \mathbb{R}_{+},
\end{aligned}
$$

and in the case $\pi(t)=t$, the asymptotically stable solutions to (1.5) are also considered. The results we obtain here are in part generalizations of those in [2], corresponding to (1.3).

\section{A fixed point theorem of Krasnosel'skii type}

Based on the Theorem 1.2 (see [1]) and [6, Theorem 3], we obtain the following theorem. The proof is similar to that of $[6$, Theorem 3]. 
Theorem 2.1. Let $\left(X,|\cdot|_{n}\right)$ be a Fréchet space and let $U, C: X \rightarrow X$ be two operators. Assume that

(i) $U$ is a $k$-contraction operator, $k \in[0,1)$ (depending on $n$ ), with respect to a family of seminorms $\|\cdot\|_{n}$ equivalent with the family $|\cdot|_{n}$;

(ii) $C$ is completely continuous;

(iii) $\lim _{|x|_{n} \rightarrow \infty}\left(|C x|_{n} /|x|_{n}\right)=0$, for all $n \in \mathbb{N}^{*}$.

Then $U+C$ has a fixed point.

Proof of Theorem 2.1. At first, we note that from the hypothesis (i), the existence and the continuity of the operator $(I-U)^{-1}$ follow. And, since a family of seminorms $\|\cdot\|_{n}$ is equivalent with the family $|\cdot|_{n}$, there exist $K_{1 n}, K_{2 n}>0$ such that

$$
K_{1 n}\|x\|_{n} \leq|x|_{n} \leq K_{2 n}\|x\|_{n}, \quad \forall n \in \mathbb{N}^{*}
$$

This implies that

(a) the set $\left\{|x|_{n}, x \in A\right\}$ is bounded if and only if $\left\{\|x\|_{n}, x \in A\right\}$ is bounded, for $A \subset X$ and for all $n \in \mathbb{N}^{*}$;

(b) for each sequence $\left(x_{m}\right)$ in $X$, for all $n \in \mathbb{N}^{*}$, since

$$
\lim _{m \rightarrow \infty}\left|x_{m}-x\right|_{n}=0 \Longleftrightarrow \lim _{m \rightarrow \infty}\left\|x_{m}-x\right\|_{n}=0
$$

$\left(x_{m}\right)$ converges to $x$ with respect to $|\cdot|_{n}$ if and only if $\left(x_{m}\right)$ converges to $x$ with respect to $\|\cdot\|_{n}$.

Consequently the condition (ii) is satisfied with respect to $\|\cdot\|_{n}$.

On the other hand, we also have

$$
\frac{K_{1 n}}{K_{2 n}} \frac{\|C x\|_{n}}{\|x\|_{n}} \leq K_{1 n} \frac{\|C x\|_{n}}{|x|_{n}} \leq \frac{|C x|_{n}}{|x|_{n}} \leq K_{2 n} \frac{\|C x\|_{n}}{|x|_{n}} \leq \frac{K_{2 n}}{K_{1 n}} \frac{\|C x\|_{n}}{\|x\|_{n}}, \quad \forall x \in X, \forall n \in \mathbb{N}^{*} .
$$

Hence, $\lim _{|x|_{n} \rightarrow \infty}\left(|C x|_{n} /|x|_{n}\right)=0$ is equivalent to $\lim _{\|x\|_{n} \rightarrow \infty}\left(\|C x\|_{n} /\|x\|_{n}\right)=0$.

Now we will prove that $U+C$ has a fixed point.

For any $a \in X$, define the operator $U_{a}: X \rightarrow X$ by $U_{a}(x)=U(x)+a$. It is easy to see that $U_{a}$ is a $k$-contraction mapping and so for each $a \in X, U_{a}$ admits a unique fixed point, it is denoted by $\phi(a)$, then

$$
U_{a}(\phi(a))=\phi(a) \Longleftrightarrow U(\phi(a))+a=\phi(a) \Longleftrightarrow \phi(a)=(I-U)^{-1}(a) .
$$

Let $u_{0}$ be a fixed point of $U$. For each $x \in X$, consider $U_{C(x)}^{m}\left(u_{0}\right), m \in \mathbb{N}^{*}$, where

$$
U_{C(x)}^{m}(y)=U_{C(x)}\left(U_{C(x)}^{m-1}(y)\right)=U\left(U_{C(x)}^{m-1}(y)\right)+C(x), \quad \forall y \in X
$$


4 On a fixed point theorem and application

We note more that for any $n \in \mathbb{N}^{*}$ being fixed, for all $m \in \mathbb{N}^{*}$,

$$
\begin{aligned}
\left\|U_{C(x)}^{m}\left(u_{0}\right)-u_{0}\right\|_{n} & =\left\|U_{C(x)}\left(U_{C(x)}^{m-1}\left(u_{0}\right)\right)-U\left(u_{0}\right)\right\|_{n} \\
& \leq\left\|U_{C(x)}\left(U_{C(x)}^{m-1}\left(u_{0}\right)\right)-U\left(U_{C(x)}^{m-1}\left(u_{0}\right)\right)\right\|_{n}+\left\|U\left(U_{C(x)}^{m-1}\left(u_{0}\right)\right)-U\left(u_{0}\right)\right\|_{n} \\
& \leq\|C(x)\|_{n}+k\left\|U_{C(x)}^{m-1}\left(u_{0}\right)-u_{0}\right\|_{n},
\end{aligned}
$$

thus, by induction, for all $m \in \mathbb{N}^{*}$, we can show that

$$
\left\|U_{C(x)}^{m}\left(u_{0}\right)-u_{0}\right\|_{n} \leq\left(1+k+\cdots+k^{m-1}\right)\|C(x)\|_{n} \leq \alpha\|C(x)\|_{n}
$$

where $\alpha=1 / 1-k>1$. By the condition (iii) satisfied with respect to $\|\cdot\|_{n}$ as above, for $1 / 4 \alpha>0$, there exists $\widetilde{M}>0$ (we choose $\widetilde{M}>\left\|u_{0}\right\|_{n}$ ) such that

$$
\|x\|_{n}>\widetilde{M} \Longrightarrow\|C x\|_{n}<\frac{1}{4 \alpha}\|x\|_{n}
$$

Choose a positive constant $r_{1 n}>\widetilde{M}+\left\|u_{0}\right\|_{n}$. Thus, for all $x \in X$, we consider the following two cases.

Case $1\left(\left\|x-u_{0}\right\|_{n}>r_{1 n}\right)$. Since $\|x\|_{n}+\left\|u_{0}\right\|_{n} \geq\left\|x-u_{0}\right\|_{n}>r_{1 n}>\widetilde{M}+\left\|u_{0}\right\|_{n} \Rightarrow\|x\|_{n}>\widetilde{M}$, we have

$$
\begin{aligned}
\|C x\|_{n} & <\frac{1}{4 \alpha}\|x\|_{n} \leq \frac{1}{4 \alpha}\left[\left\|x-u_{0}\right\|_{n}+\left\|u_{0}\right\|_{n}\right] \\
& <\frac{1}{4 \alpha}\left[\left\|x-u_{0}\right\|_{n}+\left\|x-u_{0}\right\|_{n}\right]=\frac{1}{2 \alpha}\left\|x-u_{0}\right\|_{n}
\end{aligned}
$$

Case $2\left(\left\|x-u_{0}\right\|_{n} \leq r_{1 n}\right)$. By (ii) satisfied with respect to $\|\cdot\|_{n}$, there is a positive constant $\beta$ such that

$$
\|C x\|_{n} \leq \beta
$$

Choose $r_{2 n}>\alpha \beta$. Put

$$
D_{n}=\left\{x \in X:\|x\|_{n} \leq r_{2 n}\right\}, \quad D=\bigcap_{n \in \mathbb{N}^{*}} D_{n}
$$

Then $u_{0} \in D$ and $D$ is bounded, closed, and convex in $X$.

For each $x \in D$ and for each $n \in \mathbb{N}^{*}$, as above we also consider two cases.

If $\left\|x-u_{0}\right\|_{n} \leq r_{1 n}$, then by (2.7), (2.10),

$$
\left\|U_{C(x)}^{m}\left(u_{0}\right)-u_{0}\right\|_{n} \leq \alpha\|C(x)\|_{n} \leq \alpha \beta<r_{2 n} .
$$


If $r_{1 n}<\left\|x-u_{0}\right\|_{n} \leq r_{2 n}$, then by (2.7), (2.9),

$$
\left\|U_{C(x)}^{m}\left(u_{0}\right)-\left(u_{0}\right)\right\|_{n} \leq \alpha\|C(x)\|_{n} \leq \alpha \frac{1}{2 \alpha} r_{2 n}=\frac{1}{2} r_{2 n}<r_{2 n} .
$$

We obtain $U_{C(x)}^{m}\left(u_{0}\right) \in D$ for all $x \in D$.

On the other hand, by $U_{C(x)}$ being a contraction mapping, the sequence $U_{C(x)}^{m}\left(u_{0}\right)$ converges to the unique fixed point $\phi(C(x))$ of $U_{C(x)}$, as $m \rightarrow \infty$, it implies that $\phi(C(x)) \in$ $D$, for all $x \in D$. Hence, $(I-U)^{-1} C(D) \subset D$.

Applying the Schauder fixed point theorem, the operator $(I-U)^{-1} C$ has a fixed point in $D$ that is also a fixed point of $U+C$ in $D$.

\section{Existence of solution}

Let $X=C\left(\mathbb{R}_{+}, E\right)$ be the space of all continuous functions on $\mathbb{R}_{+}$to $E$ which is equipped with the numerable family of seminorms

$$
|x|_{n}=\sup _{t \in[0, n]}\{|x(t)|\}, \quad n \geq 1 .
$$

Then $\left(X,|x|_{n}\right)$ is complete in the metric

$$
d(x, y)=\sum_{n=1}^{\infty} 2^{-n} \frac{|x-y|_{n}}{1+|x-y|_{n}}
$$

and $X$ is the Fréchet space. Consider in $X$ the other family of seminorms $\|x\|_{n}$ defined as follows:

$$
\|x\|_{n}=|x|_{\gamma_{n}}+|x|_{h_{n}}, \quad n \geq 1
$$

where

$$
|x|_{h_{n}}=\sup _{t \in\left[\gamma_{n}, n\right]}\left\{e^{-h_{n}\left(t-\gamma_{n}\right)}|x(t)|\right\}
$$

$\gamma_{n} \in(0, n)$ and $h_{n}>0$ are arbitrary numbers, which is equivalent to $|x|_{n}$, since

$$
e^{-h_{n}\left(n-\gamma_{n}\right)}|x|_{n} \leq\|x\|_{n} \leq 2|x|_{n}, \quad \forall x \in X, \forall n \geq 1 .
$$

We make the following assumptions.

$\left(\mathrm{A}_{1}\right)$ There exists a constant $L \in[0,1)$ such that

$$
|f(t, x)-f(t, y)| \leq L|x-y|, \quad \forall x, y \in E, \forall t \in \mathbb{R}_{+} .
$$

$\left(\mathrm{A}_{2}\right)$ There exists a continuous function $\omega_{1}: \Delta \rightarrow \mathbb{R}_{+}$such that

$$
|V(t, s, x)-V(t, s, y)| \leq \omega_{1}(t, s)|x-y|, \quad \forall x, y \in E, \forall(t, s) \in \Delta .
$$

$\left(\mathrm{A}_{3}\right) G$ is completely continuous such that $G(t, \cdot, \cdot): I \times J \rightarrow E$ is continuous uniformly with respect to $t$ in any bounded interval, for any bounded $I \subset[0, \infty)$ and any bounded $J \subset E$. 
6 On a fixed point theorem and application

$\left(\mathrm{A}_{4}\right)$ There exists a continuous function $\omega_{2}: \Delta \rightarrow \mathbb{R}_{+}$such that

$$
\lim _{|x| \rightarrow \infty} \frac{|G(t, s, x)|-\omega_{2}(t, s)}{|x|}=0
$$

uniformly in $(t, s)$ in any bounded subsets of $\Delta$.

Theorem 3.1. Let $\left(A_{1}\right)-\left(A_{4}\right)$ hold. Then (1.2) has a solution on $[0, \infty)$.

Proof of Theorem 3.1. The proof consists of Steps 1-4.

Step 1. In $X$, we consider the equation

$$
x(t)=q(t)+f(t, x(t)), \quad t \in \mathbb{R}_{+} .
$$

We have the following lemma.

LEMma 3.2. Let $\left(A_{1}\right)$ hold. Then (3.9) has a unique solution.

Proof. By hypothesis $\left(\mathrm{A}_{1}\right)$, the operator $\Phi: X \rightarrow X$, which is defined as follows:

$$
\Phi x(t)=q(t)+f(t, x(t)), \quad x \in X, t \in \mathbb{R}_{+}
$$

is the $L$-contraction mapping on the Fréchet space $\left(X,|x|_{n}\right)$. By applying the Banach space (see [1, Theorem B]), $\Phi$ admits a unique fixed point $\xi \in X$. The lemma is proved.

By the transformation $x=y+\xi$, we can write (1.2) in the form

$$
y(t)=A y(t)+B y(t)+C y(t), \quad t \in \mathbb{R}_{+},
$$

where

$$
\begin{gathered}
A y(t)=q(t)+f(t, y(t)+\xi(t))-\xi(t), \\
B y(t)=\int_{0}^{t} V(t, s, y(s)+\xi(s)) d s, \\
C y(t)=\int_{0}^{t} G(t, s, y(s)+\xi(s)) d s .
\end{gathered}
$$

Step 2. Put $U=A+B$. It follows from the assumptions $\left(\mathrm{A}_{1}\right),\left(\mathrm{A}_{2}\right)$ that for all $t \in \mathbb{R}_{+}$, for all $y, \tilde{y} \in X$,

$$
|U y(t)-U \tilde{y}(t)| \leq L|y(t)-\tilde{y}(t)|+\int_{0}^{t} \omega_{1}(t, s)|y(s)-\tilde{y}(s)| d s .
$$

Therefore, by a similar proof to the proof in [2, Lemma 3.1(2)], we have $U$ a $k_{n}$-contraction operator, $k_{n} \in[0,1)$ (depending on $n$ ), with respect to a family of seminorms $\|\cdot\|_{n}$. Indeed, fix an arbitrary positive integer $n \in \mathbb{N}^{*}$. 
For all $t \in\left[0, \gamma_{n}\right]$ with $\gamma_{n} \in(0, n)$ chosen later, we have

$$
\begin{aligned}
|U y(t)-U \tilde{y}(t)| & \leq L|y(t)-\tilde{y}(t)|+\int_{0}^{t} \omega_{1}(t, s)|y(s)-\tilde{y}(s)| d s \\
& \leq\left(L+\tilde{\omega}_{1 n} \gamma_{n}\right)|y-\tilde{y}|_{\gamma_{n}},
\end{aligned}
$$

where

$$
\begin{gathered}
\tilde{\omega}_{1 n}=\sup \left\{\omega_{1}(t, s):(t, s) \in \Delta_{n}\right\}, \\
\Delta_{n}=\{(t, s) \in[0, n] \times[0, n], s \leq t\} .
\end{gathered}
$$

This implies that

$$
|U y-U \tilde{y}|_{\gamma_{n}} \leq\left(L+\widetilde{\omega}_{1 n} \gamma_{n}\right)|y-\tilde{y}|_{\gamma_{n}} .
$$

For all $t \in\left[\gamma_{n}, n\right]$, similarly, we also have

$$
|U y(t)-U \tilde{y}(t)| \leq L|y(t)-\tilde{y}(t)|+\widetilde{\omega}_{1 n} \int_{0}^{\gamma_{n}}|y(s)-\tilde{y}(s)| d s+\tilde{\omega}_{1 n} \int_{\gamma_{n}}^{t}|y(s)-\tilde{y}(s)| d s .
$$

It follows from (3.17) and the inequalities

$$
0<e^{-h_{n}\left(t-\gamma_{n}\right)}<1, \quad \forall t \in\left[\gamma_{n}, n\right], h_{n}>0,
$$

$\left(h_{n}>0\right.$ is also chosen later) that

$$
\begin{aligned}
|U y(t)-U \tilde{y}(t)| e^{-h_{n}\left(t-\gamma_{n}\right) \leq} & L|y(t)-\tilde{y}(t)| e^{-h_{n}\left(t-\gamma_{n}\right)}+\tilde{\omega}_{1 n} \gamma_{n}|y-\tilde{y}|_{\gamma_{n}} \\
& +\tilde{\omega}_{1 n} \int_{\gamma_{n}}^{t}|y(s)-\tilde{y}(s)| e^{-h_{n}\left(t-\gamma_{n}\right)} d s \\
\leq & L|y-\tilde{y}|_{h_{n}}+\tilde{\omega}_{1 n} \gamma_{n}|y-\tilde{y}|_{\gamma_{n}} \\
& +\tilde{\omega}_{1 n} \int_{\gamma_{n}}^{t}|y(s)-\tilde{y}(s)| e^{-h_{n}\left(s-\gamma_{n}\right)} e^{h_{n}(s-t)} d s \\
\leq & L|y-\tilde{y}|_{h_{n}}+\tilde{\omega}_{1 n} \gamma_{n}|y-\tilde{y}|_{\gamma_{n}}+\tilde{\omega}_{1 n}|y-\tilde{y}|_{h_{n}} \int_{\gamma_{n}}^{t} e^{h_{n}(s-t)} d s \\
\leq & L|y-\tilde{y}|_{h_{n}}+\tilde{\omega}_{1 n} \gamma_{n}|y-\tilde{y}|_{\gamma_{n}}+\frac{\tilde{\omega}_{1 n}}{h_{n}}|y-\tilde{y}|_{h_{n} .}
\end{aligned}
$$

We get

$$
|U y-U \tilde{y}|_{h_{n}} \leq\left(L+\frac{\tilde{\omega}_{1 n}}{h_{n}}\right)|y-\tilde{y}|_{h_{n}}+\tilde{\omega}_{1 n} \gamma_{n}|y-\tilde{y}|_{\gamma_{n}} .
$$


Combining (3.16)-(3.20), we deduce that

$$
\|U y-U \tilde{y}\|_{n} \leq\left(L+2 \gamma_{n} \widetilde{\omega}_{1 n}\right)|y-\tilde{y}|_{\gamma_{n}}+\left(L+\frac{\widetilde{\omega}_{1 n}}{h_{n}}\right)|y-\tilde{y}|_{h_{n}} \leq k_{n}\|y-\tilde{y}\|_{n},
$$

where $k_{n}=\max \left\{L+2 \gamma_{n} \widetilde{\omega}_{1 n}, L+\widetilde{\omega}_{1 n} / h_{n}\right\}$. Choose

$$
0<\gamma_{n}<\min \left\{\frac{1-L}{2 \widetilde{\omega}_{1 n}}, n\right\}, \quad h_{n}>\frac{\widetilde{\omega}_{1 n}}{1-L},
$$

then we have $k_{n}<1$, by (3.21), $U$ is a $k_{n}$-contraction operator with respect to a family of seminorms $\|\cdot\|_{n}$.

Step 3. We show that $C: X \rightarrow X$ is completely continuous. We first show that $C$ is continuous. For any $y_{0} \in X$, let $\left(y_{m}\right)_{m}$ be a sequence in $X$ such that $\lim _{m \rightarrow \infty} y_{m}=y_{0}$.

Let $n \in \mathbb{N}^{*}$ be fixed. Put $K=\left\{\left(y_{m}+\xi\right)(s): s \in[0, n], m \in \mathbb{N}\right\}$. Then $K$ is compact in $E$. Indeed, let $\left(\left(y_{m_{i}}+\xi\right)\left(s_{i}\right)\right)_{i}$ be a sequence in $K$. We can assume that $\lim _{i \rightarrow \infty} s_{i}=s_{0}$ and that $\lim _{i \rightarrow \infty} y_{m_{i}}+\xi=y_{0}+\xi$. We have

$$
\begin{aligned}
\left|\left(y_{m_{i}}+\xi\right)\left(s_{i}\right)-\left(y_{0}+\xi\right)\left(s_{0}\right)\right| & \leq\left|\left(y_{m_{i}}+\xi\right)\left(s_{i}\right)-\left(y_{0}+\xi\right)\left(s_{i}\right)\right|+\left|\left(y_{0}+\xi\right)\left(s_{i}\right)-\left(y_{0}+\xi\right)\left(s_{0}\right)\right| \\
& \leq\left|y_{m_{i}}-y_{0}\right|_{n}+\left|\left(y_{0}+\xi\right)\left(s_{i}\right)-\left(y_{0}+\xi\right)\left(s_{0}\right)\right|
\end{aligned}
$$

which shows that $\lim _{i \rightarrow \infty}\left(y_{m_{i}}+\xi\right)\left(s_{i}\right)=\left(y_{0}+\xi\right)\left(s_{0}\right)$ in $E$. It means that $K$ is compact in $E$. For any $\epsilon>0$, since $G$ is continuous on the compact set $[0, n] \times[0, n] \times K$, there exists $\delta>0$ such that for every $u, v \in K,|u-v|<\delta$,

$$
|G(t, s, u)-G(t, s, v)|<\frac{\epsilon}{n}, \quad \forall s, t \in[0, n] .
$$

Since $\lim _{m \rightarrow \infty} y_{m}=y_{0}$, there exists $m_{0}$ such that for $m>m_{0}$,

$$
\left|\left(y_{m}+\xi\right)(s)-\left(y_{0}+\xi\right)(s)\right|=\left|y_{m}(s)-y_{0}(s)\right|<\delta, \quad \forall s \in[0, n] .
$$

This implies that for all $t \in[0, n]$, for all $m>m_{0}$,

$$
\left|C y_{m}(t)-C y_{0}(t)\right| \leq \int_{0}^{t}\left|G\left(t, s,\left(y_{m}+\xi\right)(s)\right)-G\left(t, s,\left(y_{0}+\xi\right)(s)\right)\right| d s<\epsilon,
$$

so $\left|C y_{m}-C y_{0}\right|_{n}<\epsilon$, for all $m>m_{0}$, and the continuity of $C$ is proved.

It remains to show that $C$ maps bounded sets into relatively compact sets. Let us recall the following condition for the relative compactness of a subset in $X$.

Lemma 3.3 (see [7, Proposition 1]). Let $X=C\left(\mathbb{R}_{+}, E\right)$ be the Fréchet space defined as above and let $A$ be a subset of $X$. For each $n \in \mathbb{N}^{*}$, let $X_{n}=C([0, n], E)$ be the Banach space of all continuous functions $u:[0, n] \rightarrow E$, with the norm $\|u\|=\sup _{t \in[0, n]}\{|u(t)|\}$, and $A_{n}=$ $\left\{\left.x\right|_{[0, n]}: x \in A\right\}$.

The set $A$ in $X$ is relatively compact if and only if for each $n \in \mathbb{N}^{*}, A_{n}$ is equicontinuous in $X_{n}$ and for every $s \in[0, n]$, the set $A_{n}(s)=\left\{x(s): x \in A_{n}\right\}$ is relatively compact in $E$. 
This proposition was stated in [7] and without proving in detail. Let us prove it in the appendix. The proof follows from the Ascoli-Arzela theorem (see [5]):

Let $E$ be a Banach space with the norm $|\cdot|$ and let $\widetilde{S}$ be a compact metric space. Let $C_{E}(\tilde{S})$ be the Banach space of all continuous maps from $\tilde{S}$ to $E$ with the norm

$$
\|x\|=\sup \{|x(s)|, s \in \widetilde{S}\} .
$$

The set $A$ in $C_{E}(\widetilde{S})$ is relatively compact if and only if $A$ is equicontinuous and for every $s \in \widetilde{S}$, the set $A(s)=\{x(s): x \in A\}$ is relatively compact in $E$.

Now, let $\Omega$ be a bounded subset of $X$. We have to prove that for $n \in \mathbb{N}^{*}$, we have the following.

(a) The set $(C \Omega)_{n}$ is equicontinuous in $X_{n}$.

Put $S=\{(y+\xi)(s): y \in \Omega, s \in[0, n]\}$. Then $S$ is bounded in $E$. Since $G$ is completely continuous, the set $G\left([0, n]^{2} \times S\right)$ is relatively compact in $E$, and so $G\left([0, n]^{2} \times S\right)$ is bounded. Consequently, there exists $M_{n}>0$ such that

$$
|G(t, s,(y+\xi)(s))| \leq M_{n}, \quad \forall t, s \in[0, n], \forall y \in \Omega .
$$

For any $y \in \Omega$, for all $t_{1}, t_{2} \in[0, n]$,

$$
\begin{aligned}
\left|C y\left(t_{1}\right)-C y\left(t_{2}\right)\right|= & \left|\int_{0}^{t_{1}} G\left(t_{1}, s,(y+\xi)(s)\right) d s-\int_{0}^{t_{2}} G\left(t_{2}, s,(y+\xi)(s)\right) d s\right| \\
\leq & \int_{0}^{t_{1}}\left|G\left(t_{1}, s,(y+\xi)(s)\right)-G\left(t_{2}, s,(y+\xi)(s)\right)\right| d s \\
& +\int_{t_{1}}^{t_{2}}\left|G\left(t_{2}, s,(y+\xi)(s)\right)\right| d s .
\end{aligned}
$$

By the hypothesis $\left(A_{3}\right)$ and (3.28), the inequality (3.29) shows that $(C \Omega)_{n}$ is equicontinuous on $X_{n}$.

(b) For every $t \in[0, n]$, the set $(C \Omega)_{n}(t)=\left\{\left.C y\right|_{[0, n]}(t): y \in \Omega\right\}$ is relatively compact in $E$.

As above, the set $G\left([0, n]^{2} \times S\right)$ is relatively compact in $E$, it implies that $\overline{G\left([0, n]^{2} \times S\right)}$ is compact in $E$, and so is $\overline{\operatorname{conv}} G\left([0, n]^{2} \times S\right)$, where $\overline{\operatorname{conv}} G\left([0, n]^{2} \times S\right)$ is the convex closure of $G\left([0, n]^{2} \times S\right)$.

For every $t \in[0, n]$, for all $y \in \Omega$, it follows from

$$
\begin{gathered}
G(t, s,(y+\xi)(s)) \in G\left([0, n]^{2} \times S\right), \quad \forall s \in[0, n], \\
C y(t)=\int_{0}^{t} G(t, s,(y+\xi)(s)) d s
\end{gathered}
$$

that

$$
\overline{(C \Omega)_{n}(t)} \subset t \overline{\operatorname{conv}} G\left([0, n]^{2} \times S\right) .
$$

Hence the set $(C \Omega)_{n}(t)$ is relatively compact in $E$. 
By Lemma 3.3, $C(\Omega)$ is relatively compact in $X$. Therefore, $C$ is completely continuous. Step 3 is proved.

Step 4. Finally, we show that for all $n \in \mathbb{N} *$,

$$
\lim _{|y|_{n} \rightarrow \infty} \frac{|C y|_{n}}{|y|_{n}}=0 .
$$

For any given $\epsilon>0$, the assumption $\left(\mathrm{A}_{4}\right)$ implies that there exists $\eta>0$ such that for all $u$ with $|u|>\eta$,

$$
|G(t, s, u)|<\tilde{\omega}_{2 n}+\frac{\epsilon}{4 n}|u|, \quad \forall t, s \in[0, n],
$$

where $\widetilde{\omega}_{2 n}=\sup \left\{\omega_{2}(t, s): t, s \in[0, n]\right\}$.

On the other hand, $G$ is completely continuous, there exists $\rho>0$ such that for all $u$ with $|u| \leq \eta$,

$$
|G(t, s, u)| \leq \rho, \quad \forall t, s \in[0, n] .
$$

Combining (3.33), (3.34), for all $t, s \in[0, n]$, for all $u \in E$, we get

$$
|G(t, s, u)| \leq \rho+\tilde{\omega}_{2 n}+\frac{\epsilon}{4 n}|u| .
$$

This implies that for all $t \in[0, n]$,

$$
\begin{aligned}
|C y(t)| & \leq \int_{0}^{t}|G(t, s,(y+\xi)(s))| d s \\
& \leq n\left[\rho+\widetilde{\omega}_{2 n}+\frac{\epsilon}{4 n}\left(|y|_{n}+|\xi|_{n}\right)\right] . \\
& =n \rho+n \tilde{\omega}_{2 n}+\frac{\epsilon}{4}|\xi|_{n}+\frac{\epsilon}{4}|y|_{n} .
\end{aligned}
$$

It follows that if we choose $\mu_{n}>\max \left\{4 n \rho / \epsilon, 4 n \tilde{\omega}_{2 n} / \epsilon,|\xi|_{n}\right\}$, then for $|y|_{n}>\mu_{n}$, we have $|C y|_{n} /|y|_{n}<\epsilon$, this means that

$$
\lim _{|y|_{n} \rightarrow \infty} \frac{|C y|_{n}}{|y|_{n}}=0 .
$$

By applying Theorem 2.1, the operator $U+C$ has a fixed point $y$ in $X$. Then (1.2) has a solution $x=y+\xi$ on $[0, \infty)$. Theorem 3.1 is proved.

\section{The asymptotically stable solutions}

We now consider the asymptotically stable solutions for (1.2) defined as follows.

Definition 4.1. A function $x$ is said to be an asymptotically stable solution of (1.2) if for any solution $\tilde{x}$ of (1.2),

$$
\lim _{t \rightarrow \infty}|x(t)-\tilde{x}(t)|=0
$$


In this section, we assume that $\left(\mathrm{A}_{1}\right)-\left(\mathrm{A}_{4}\right)$ hold and assume in addition that

$\left(\mathrm{A}_{5}\right) V(t, s, 0)=0$, for all $(t, s) \in \Delta$;

$\left(\mathrm{A}_{6}\right)$ there exist two continuous functions $\omega_{3}, \omega_{4}: \Delta \rightarrow \mathbb{R}_{+}$such that

$$
|G(t, s, x)| \leq \omega_{3}(t, s)+\omega_{4}(t, s)|x|, \quad \forall(t, s) \in \Delta .
$$

Then, by Theorem 3.1, (1.2) has a solution on $(0, \infty)$.

On the other hand, if $x$ is a solution of (1.2) then, as Step 1 of the proof of Theorem 3.1, $y=x-\xi$ satisfies (3.11). This implies that for all $t \in \mathbb{R}_{+}$,

$$
|y(t)| \leq|A y(t)|+|B y(t)|+|C y(t)|,
$$

where

$$
\begin{aligned}
& A y(t)=q(t)+f(t, y(t)+\xi(t))-\xi(t), \quad A 0=0, \\
& B y(t)=\int_{0}^{t} V(t, s, y(s)+\xi(s)) d s, \quad \text { in which } V(t, s, 0)=0, \\
& C y(t)=\int_{0}^{t} G(t, s, y(s)+\xi(s)) d s .
\end{aligned}
$$

Consequently, for all $t \in \mathbb{R}_{+}$,

$$
|y(t)| \leq L|y(t)|+\int_{0}^{t} \omega_{1}(t, s)|y(s)+\xi(s)| d s+\int_{0}^{t}\left[\omega_{3}(t, s)+\omega_{4}(t, s)|y(s)+\xi(s)|\right] d s .
$$

It follows that

$$
|y(t)| \leq \frac{1}{1-L} \int_{0}^{t} \omega(t, s)|y(s)| d s+a(t)
$$

where

$$
\begin{gathered}
\omega(t, s)=\omega_{1}(t, s)+\omega_{4}(t, s) \\
a(t)=\frac{1}{1-L} \int_{0}^{t} \omega(t, s)|\xi(s)| d s+\frac{1}{1-L} \int_{0}^{t} \omega_{3}(t, s) d s .
\end{gathered}
$$

Using the inequality $(a+b)^{2} \leq 2\left(a^{2}+b^{2}\right)$, for all $a, b \in \mathbb{R}$, we get

$$
|y(t)|^{2} \leq \frac{2}{(1-L)^{2}} \int_{0}^{t} \omega^{2}(t, s) d s \int_{0}^{t}|y(s)|^{2} d s+2 a^{2}(t) .
$$

Putting $v(t)=|y(t)|^{2}, b(t)=\left(2 /(1-L)^{2}\right) \int_{0}^{t} \omega^{2}(t, s) d s,(4.8)$ is rewritten as follows:

$$
v(t) \leq b(t) \int_{0}^{t} v(s) d s+2 a^{2}(t)
$$


By (4.9), based on classical estimates, we obtain

$$
|y(t)|^{2}=v(t) \leq 2 a^{2}(t)+b(t) e^{\int_{0}^{t} b(s) d s} \int_{0}^{t} 2 e^{-\int_{0}^{s} b(u) d u} a^{2}(s) d s, \quad \forall t \in \mathbb{R}_{+} .
$$

Then we have the following theorem about the asymptotically stable solutions.

Theorem 4.2. Let $\left(A_{1}\right)-\left(A_{6}\right)$ hold. If

$$
\lim _{t \rightarrow \infty} 2 a^{2}(t)+b(t) e^{\int_{0}^{t} b(s) d s} \int_{0}^{t} 2 e^{-\int_{0}^{s} b(u) d u} a^{2}(s) d s=0
$$

where

$$
\begin{gathered}
a(t)=\frac{1}{1-L} \int_{0}^{t}\left[\omega_{1}(t, s)+\omega_{4}(t, s)\right]|\xi(s)| d s+\frac{1}{1-L} \int_{0}^{t} \omega_{3}(t, s) d s, \\
b(t)=\frac{2}{(1-L)^{2}} \int_{0}^{t}\left[\omega_{1}(t, s)+\omega_{4}(t, s)\right]^{2} d s,
\end{gathered}
$$

then every solution $x$ to (1.2) is an asymptotically stable solution.

Furthermore,

$$
\lim _{t \rightarrow \infty}|x(t)-\xi(t)|=0
$$

Proof of Theorem 4.2. Let $x, \tilde{x}$ be two solutions to (1.2).

Then $y=x-\xi, \tilde{y}=\tilde{x}-\xi$ are solutions to (3.11). It follows from (4.10) that

$$
|y(t)|^{2} \leq 2 a^{2}(t)+b(t) e^{\int_{0}^{t} b(s) d s} \int_{0}^{t} 2 e^{-\int_{0}^{s} b(u) d u} a^{2}(s) d s
$$

for all $t \in \mathbb{R}_{+}$, and so is $|\tilde{y}(t)|^{2}$.

It follows from (4.11) and (4.14) that

$$
\lim _{t \rightarrow \infty}|x(t)-\xi(t)|=0
$$

Put $c(t)=2 a^{2}(t)+b(t) e^{\int_{0}^{t} b(s) d s} \int_{0}^{t} 2 e^{-\int_{0}^{s} b(u) d u} a^{2}(s) d s$. Then, by (4.14),

$$
|x(t)-\tilde{x}(t)|=|y(t)-\tilde{y}(t)| \leq 2 \sqrt{c(t)}, \quad \forall t \in \mathbb{R}_{+}
$$

Combining (4.11), (4.16),

$$
\lim _{t \rightarrow \infty}|x(t)-\tilde{x}(t)|=0 .
$$

Theorem 4.2 is proved. 
Remark 4.3. We present an example when condition (4.11) holds.

Let the following assumptions hold:

$\left(\mathrm{H}_{1}\right) \int_{0}^{+\infty}|q(s)|^{2} d s<+\infty, \int_{0}^{+\infty}|f(s, 0)|^{2} d s<+\infty$;

$\left(\mathrm{H}_{2}\right) \lim _{t \rightarrow \infty} \int_{0}^{t} \omega_{3}(t, s) d s=0, \int_{0}^{+\infty}\left[\int_{0}^{s} \omega_{3}(s, u) d u\right]^{2} d s<+\infty$;

$\left(\mathrm{H}_{3}\right)$ there exist continuous functions $g_{i}, h_{i}: \mathbb{R}_{+} \rightarrow \mathbb{R}_{+}, i=1,4$ such that for $i=1,4$,

(i) $\omega_{i}(t, s)=g_{i}(t) h_{i}(s)$, for all $(t, s) \in \Delta$,

(ii) $\lim _{t \rightarrow \infty} g_{i}(t)=0$,

(iii) $\int_{0}^{+\infty} g_{i}^{2}(s) d s<+\infty, \int_{0}^{+\infty} h_{i}^{2}(s) d s<+\infty$.

Then condition (4.11) holds. Indeed, we have the following.

Since $\xi$ is a (unique) fixed point of $\Phi$, for all $t \in \mathbb{R}^{+}$, we have

$$
\begin{aligned}
|\xi(t)| & \leq|q(t)|+|f(t, \xi(t))| \leq|q(t)|+|f(t, 0)|+|f(t, \xi(t))-f(t, 0)| \\
& \leq|q(t)|+|f(t, 0)|+L|\xi(t)| .
\end{aligned}
$$

This means that

$$
|\xi(t)| \leq \frac{1}{1-L}(|q(t)|+|f(t, 0)|)
$$

so

$$
|\xi(t)|^{2} \leq \frac{2}{(1-L)^{2}}\left(|q(t)|^{2}+|f(t, 0)|^{2}\right)
$$

and hence $\int_{0}^{+\infty}|\xi(s)|^{2} d s<+\infty$, by the hypothesis $\left(\mathrm{H}_{1}\right)$.

Therefore, it follows from $\left(\mathrm{H}_{3}\right)$ that

$$
\begin{gathered}
\left(\int_{0}^{+\infty} h_{i}(s)|\xi(s)| d s\right)^{2} \leq \int_{0}^{+\infty} h_{i}^{2}(s) d s \int_{0}^{+\infty}|\xi(s)|^{2} d s<+\infty, \quad i=1,4 \\
\lim _{t \rightarrow \infty} \int_{0}^{t} \omega_{i}(t, s)|\xi(s)| d s=\lim _{t \rightarrow \infty} g_{i}(t) \int_{0}^{t} h_{i}(s)|\xi(s)| d s=0, \quad i=1,4 .
\end{gathered}
$$

Combining these and $\left(\mathrm{H}_{2}\right)$, we obtain

$$
a(t)=\frac{1}{1-L} \int_{0}^{t} \omega_{1}(t, s)|\xi(s)| d s+\frac{1}{1-L} \int_{0}^{t}\left[\omega_{3}(t, s)+\omega_{4}(t, s)|\xi(s)|\right] d s \longrightarrow 0
$$

as $t \rightarrow \infty$.

By $\left(\mathrm{H}_{3}\right)$, we also have

$$
\begin{aligned}
\int_{0}^{t} \omega^{2}(t, s) d s & \leq 2 \int_{0}^{t}\left[\omega_{1}^{2}(t, s)+\omega_{2}^{2}(t, s)\right] d s \\
& =2 g_{1}^{2}(t) \int_{0}^{t} h_{1}^{2}(s) d s+2 g_{4}^{2}(t) \int_{0}^{t} h_{4}^{2}(s) d s \longrightarrow 0, \text { as } t \longrightarrow \infty,
\end{aligned}
$$


14 On a fixed point theorem and application

and it follows that

$$
b(t)=\frac{2}{(1-L)^{2}} \int_{0}^{t} \omega^{2}(t, s) d s \longrightarrow 0, \quad \text { as } t \longrightarrow \infty .
$$

Furthermore, it follows from $(4.23)$ and $\left(\mathrm{H}_{3}\right)$ (iii) that

$$
\int_{0}^{+\infty} b(s) d s<+\infty
$$

On the other hand, by

$$
\begin{aligned}
a^{2}(t) \leq & \frac{3}{(1-L)^{2}} g_{1}^{2}(t) \int_{0}^{t} h_{1}^{2}(s) d s \int_{0}^{t}|\xi(s)|^{2} d s+\frac{3}{(1-L)^{2}}\left[\int_{0}^{t} \omega_{3}(t, s) d s\right]^{2} \\
& +\frac{3}{(1-L)^{2}} g_{4}^{2}(t) \int_{0}^{t} h_{4}^{2}(s) d s \int_{0}^{t}|\xi(s)|^{2} d s
\end{aligned}
$$

$\left(\mathrm{H}_{2}\right)$ and $\left(\mathrm{H}_{3}\right)(\mathrm{iii})$, we get

$$
\int_{0}^{+\infty} a^{2}(s) d s<+\infty
$$

Hence, from (4.22), (4.24)-(4.27), we conclude that

$$
\lim _{t \rightarrow \infty} 2 a^{2}(t)+b(t) e^{\int_{0}^{t} b(s) d s} \int_{0}^{t} 2 e^{-\int_{0}^{s} b(u) d u} a^{2}(s) d s=0 .
$$

Remark 4.4. If $g_{i}: \mathbb{R}_{+} \rightarrow \mathbb{R}_{+}, i=1,4$, is uniformly continuous, then the hypothesis $\left(\mathrm{H}_{3}\right)(\mathrm{ii})$, $\lim _{t \rightarrow \infty} g_{i}(t)=0$, follows from the hypothesis $\left(\mathrm{H}_{3}\right)(\mathrm{iii})_{1}, \int_{0}^{+\infty} g_{i}^{2}(s) d s<+\infty$.

Remark 4.5 (an example). Let us give the following illustrated example for the results we obtain as above.

Let $E=C([0,1], \mathbb{R})$ with the usual norm $\|u\|=\sup _{\zeta \in[0,1]}\{|u(\zeta)|\}$.

Consider (1.2), where

$$
\begin{array}{rlrl}
q: \mathbb{R}_{+} \longrightarrow E, & t \longmapsto q(t), \\
f: \mathbb{R}_{+} \times E \longrightarrow E, & (t, x) & \longmapsto f(t, x), \\
V: \Delta \times E \longrightarrow E, & (t, s, x) & \longmapsto V(t, s, x), \\
G: \Delta \times E \longrightarrow E, & (t, s, x) & \longmapsto G(t, s, x),
\end{array}
$$


such that for every $x \in X=C\left(\mathbb{R}_{+}, E\right)$, for all $t, s \geq 0(s \leq t)$, for all $\zeta \in[0,1]$,

$$
\begin{gathered}
q(t)(\zeta) \equiv q(t, \zeta)=\frac{1-k}{e^{t}+\zeta} e^{-2 t}, \\
f(t, x)(\zeta)=\frac{k}{e^{t}+\zeta} e^{-2 t} \sin \left[\frac{\pi}{2}\left(e^{t}+\zeta\right) x(\zeta)\right], \\
V(t, s, x)(\zeta)=\frac{1}{e^{t}+\zeta} e^{-2 s}\left(e^{s}+\zeta\right)|x(\zeta)|, \\
G(t, s, x)(\zeta)=\frac{1}{e^{t}+\zeta} e^{-2 s} \sqrt{e^{s}} \sqrt{\|x\|},
\end{gathered}
$$

in which $k<2 / \pi$ is a positive constant.

We first note that for every $x, y \in X=C\left(\mathbb{R}_{+}, E\right)$, for all $t, s \geq 0(s \leq t)$, and for all $\zeta \in$ $[0,1]$

$$
\begin{aligned}
|f(t, x)(\zeta)-f(t, y)(\zeta)| & \leq \frac{k}{e^{t}+\zeta} e^{-2 t}\left|\sin \left[\frac{\pi}{2}\left(e^{t}+\zeta\right) x(\zeta)\right]-\sin \left[\frac{\pi}{2}\left(e^{t}+\zeta\right) y(\zeta)\right]\right| \\
& \leq k e^{-2 t} \frac{\pi}{2}|x(\zeta)-y(\zeta)| \leq k \frac{\pi}{2}\|x-y\|, \\
|G(t, s, x)(\zeta)| & =\frac{1}{e^{t}+\zeta} e^{-2 s} \sqrt{e^{s}} \sqrt{\|x\|} \\
& \leq \frac{1}{2\left(e^{t}+\zeta\right)} e^{-2 s} \sqrt{e^{s}}+\frac{1}{2\left(e^{t}+\zeta\right)} e^{-2 s} \sqrt{e^{s}}\|x\|,
\end{aligned}
$$

by Cauchy's inequality.

Combining these and the given hypotheses as above, we have $q, f, V, G$ satisfying $\left(A_{1}\right)-\left(A_{6}\right)$, with

$$
\begin{gathered}
\omega_{1}(t, s)=e^{-t} e^{-2 s}\left(e^{s}+1\right), \quad \omega_{2}(t, s)=0, \\
\omega_{3}(t, s)=\omega_{4}(t, s)=\frac{1}{2} e^{-t} e^{-2 s} \sqrt{e^{s}} .
\end{gathered}
$$

Furthermore, it is obvious that $\left(\mathrm{H}_{1}\right)-\left(\mathrm{H}_{3}\right)$ hold.

We conclude that Theorems 3.1, 4.2 hold for (1.2), in this case.

For more details, let us consider a solution $x(t)$ of (1.2) as follows.

Let $x \in X=C\left(\mathbb{R}_{+}, E\right)$ such that for all $t \in \mathbb{R}_{+}$,

$$
x(t)(\zeta) \equiv x(t, \zeta)=\frac{1}{e^{t}+\zeta}, \quad \forall \zeta \in[0,1]
$$

It is clear that $x$ defined as above is the solution of (1.2). Moreover,

$$
\|x(t)\|=\sup _{\zeta \in[0,1]}\left\{\left|\frac{1}{e^{t}+\zeta}\right|\right\}=e^{-t} \longrightarrow 0, \quad \text { as } t \longrightarrow+\infty .
$$


On the other hand, by

$$
|f(t, x(t))(\zeta)-f(t, y(t))(\zeta)| \leq k \frac{\pi}{2}\|x(t)-y(t)\|,
$$

for all $x, y \in X$, for all $t \in \mathbb{R}_{+}$, and for all $\zeta \in[0,1]$, we obtain

$$
\sup _{t \in[0, n]}\{\|f(t, x(t))-f(t, y(t))\|\} \leq k \frac{\pi}{2} \sup _{t \in[0, n]}\{\|x(t)-y(t)\|\},
$$

for all $n \in \mathbb{N}^{*}$. Thus the equation

$$
x(t)=q(t)+f(t, x(t)), \quad t \geq 0
$$

has a unique $\xi(t) \in X$. We see at once that for all $\zeta \in[0,1]$,

$$
\begin{aligned}
|\xi(t, \zeta)| & \leq|q(t, \zeta)|+|f(t, \xi(t))(\zeta)| \leq \frac{1-k}{e^{t}+\zeta} e^{-2 t}+\frac{k}{e^{t}+\zeta} e^{-2 t}\left|\sin \left[\frac{\pi}{2}\left(e^{t}+\zeta\right) \xi(t, \zeta)\right]\right| \\
& \leq(1-k) e^{-3 t}+k e^{-3 t}=e^{-3 t}
\end{aligned}
$$

This implies that

$$
\|x(t)-\xi(t)\| \leq e^{-t}+e^{-3 t} .
$$

Therefore, $\lim _{t \rightarrow \infty}\|x(t)-\xi(t)\|=0$.

\section{The general case}

Since this will cause no confusion, let us use the same letters $V, G, \omega_{i}, i=1,2,3,4 ; \Phi, \xi$, $A, B, C, U$ to define the functions of Section 3 and of this section, respectively.

We consider the following equation:

$$
\begin{aligned}
x(t)= & q(t)+\hat{f}(t, x(t), x(\pi(t))) \\
& +\int_{0}^{t} V(t, s, x(s), x(\sigma(s))) d s+\int_{0}^{t} G(t, s, x(s), x(\chi(s))) d s, \quad t \in \mathbb{R}_{+},
\end{aligned}
$$

where $q: \mathbb{R}_{+} \rightarrow E ; \hat{f}: \mathbb{R}_{+} \times E \times E \rightarrow E ; G, V: \Delta \times E \times E \rightarrow E$ are supposed to be continuous and $\Delta=\left\{(t, s) \in \mathbb{R}_{+} \times \mathbb{R}_{+}, s \leq t\right\}$, the functions $\pi, \sigma, \chi: \mathbb{R}_{+} \rightarrow \mathbb{R}_{+}$are continuous.

We make the following assumptions.

$\left(\mathrm{I}_{1}\right)$ There exists a constant $L \in[0,1)$ such that

$$
|\hat{f}(t, x, u)-\hat{f}(t, y, v)| \leq \frac{L}{2}(|x-y|+|u-v|), \quad \forall x, y, u, v \in E, \forall t \in \mathbb{R}_{+} .
$$

$\left(\mathrm{I}_{2}\right)$ There exists a continuous function $\omega_{1}: \Delta \rightarrow \mathbb{R}_{+}$such that

$$
|V(t, s, x, u)-V(t, s, y, v)| \leq \omega_{1}(t, s)(|x-y|+|u-v|), \quad \forall x, y, u, v \in E, \forall(t, s) \in \Delta .
$$


$\left(\mathrm{I}_{3}\right) G$ is completely continuous such that $G(t, \cdot, \cdot, \cdot \cdot): I \times J_{1} \times J_{2} \rightarrow E$ is continuous uniformly with respect to $t$ in any bounded interval, for any bounded subset $I \subset[0, \infty)$ and for any bounded subset $J_{1}, J_{2} \subset E$.

$\left(\mathrm{I}_{4}\right)$ There exists a continuous function $\omega_{2}: \Delta \rightarrow \mathbb{R}_{+}$such that

$$
\lim _{|x|+|u| \rightarrow \infty} \frac{|G(t, s, x, u)|-\omega_{2}(t, s)}{|x|+|u|}=0
$$

uniformly in $(t, s)$ in any bounded subsets of $\Delta$.

$\left(\mathrm{I}_{5}\right) 0<\pi(t) \leq t, 0<\sigma(t) \leq t, \chi(t) \leq t$, for all $t \in \mathbb{R}_{+}$.

Theorem 5.1. Let $\left(I_{1}\right)-\left(I_{5}\right)$ hold. Then (5.1) has a solution on $(0, \infty)$.

Proof of Theorem 5.1. These follow by the same method as in Section 3. However, there are also some changes.

At first, we note that the following exist. (a) By hypothesis $\left(\mathrm{I}_{1}\right)$ and $0<\pi(t) \leq t$, for all $t \in \mathbb{R}_{+}$, the operator $\Phi: X \rightarrow X$ defined by

$$
\Phi x(t)=q(t)+\hat{f}(t, x(t), x(\pi(t))), \quad \forall x \in X, t \in \mathbb{R}_{+},
$$

is the $L$-contraction mapping on the Fréchet space $\left(X,|x|_{n}\right)$. Indeed, fix $n \in \mathbb{N}^{*}$. For all $x \in X$ and for all $t \in[0, n]$,

$$
\begin{aligned}
|\Phi x(t)-\Phi y(t)| & \leq \frac{L}{2}(|x(t)-y(t)|+|x(\pi(t))-y(\pi(t))|) \\
& \leq \frac{L}{2}\left(|x-y|_{n}+|x-y|_{n}\right)=L|x-y|_{n} .
\end{aligned}
$$

So $|\Phi x-\Phi y|_{n} \leq L|x-y|_{n}$. Therefore, $\Phi$ admits a unique fixed point $\xi \in X$.

By the transformation $x=y+\xi,(5.1)$ is rewritten as follows:

$$
y(t)=A y(t)+B y(t)+C y(t), \quad t \in \mathbb{R}_{+},
$$

where

$$
\begin{gathered}
A y(t)=q(t)+\hat{f}(t, y(t)+\xi(t), y(\pi(t))+\xi(\pi(t)))-\xi(t), \quad A 0=0, \\
B y(t)=\int_{0}^{t} V(t, s, y(s)+\xi(s), y(\sigma(t))+\xi(\sigma(t))) d s, \\
C y(t)=\int_{0}^{t} G(t, s, y(s)+\xi(s), y(\chi(t))+\xi(\chi(t))) d s .
\end{gathered}
$$

(b) Put $U=A+B$. Then, $U$ is a contraction operator with respect to a family of seminorms $\|\cdot\|_{n}$. Indeed, fix an arbitrary positive integer $n \in \mathbb{N}^{*}$. 
For all $t \in\left[0, \gamma_{n}\right]$ with $\gamma_{n} \in(0, n), \gamma_{n}<\hat{\sigma}_{n}=\min \{\sigma(t), t \in[0, n]\}, \gamma_{n}<\hat{\pi}_{n}=\min \{\pi(t)$, $t \in[0, n]\}$ chosen later, we have

$$
\begin{aligned}
|U y(t)-U \tilde{y}(t)| \leq & \frac{L}{2}|y(t)-\tilde{y}(t)|+\frac{L}{2}|y(\pi(t))-\tilde{y}(\pi(t))| \\
& +\int_{0}^{t} \omega_{1}(t, s)(|y(s)-\tilde{y}(s)|+|y(\sigma(s))-\tilde{y}(\sigma(s))|) d s \\
\leq & \left(L+2 \widetilde{\omega}_{1 n} \gamma_{n}\right)|y-\tilde{y}|_{\gamma_{n} .}
\end{aligned}
$$

This implies that

$$
|U y-U \tilde{y}|_{\gamma_{n}} \leq\left(L+2 \widetilde{\omega}_{1 n} \gamma_{n}\right)|y-\tilde{y}|_{\gamma_{n}} .
$$

For all $t \in\left[\gamma_{n}, n\right]$, similarly, we also have

$$
\begin{aligned}
|U y(t)-U \tilde{y}(t)| \leq & \frac{L}{2}|y(t)-\tilde{y}(t)|+\frac{L}{2}|y(\pi(t))-\tilde{y}(\pi(t))| \\
& +\tilde{\omega}_{1 n} \int_{0}^{\gamma_{n}}(|y(s)-\tilde{y}(s)|+|y(\sigma(s))-\tilde{y}(\sigma(s))|) d s \\
& +\widetilde{\omega}_{1 n} \int_{\gamma_{n}}^{t}(|y(s)-\tilde{y}(s)|+|y(\sigma(s))-\tilde{y}(\sigma(s))|) d s .
\end{aligned}
$$

By the inequalities

$$
\begin{aligned}
& 0<e^{-h_{n}\left(t-\gamma_{n}\right)}<e^{-h_{n}\left(\pi(t)-\gamma_{n}\right)}<1, \quad \forall t \in\left[\gamma_{n}, n\right], \\
& 0<e^{-h_{n}\left(t-\gamma_{n}\right)}<e^{-h_{n}\left(\sigma(t)-\gamma_{n}\right)}<1, \quad \forall t \in\left[\gamma_{n}, n\right],
\end{aligned}
$$

in which $h_{n}>0$ is also chosen later, we get

$$
\begin{aligned}
& |U y(t)-U \tilde{y}(t)| e^{-h_{n}\left(t-\gamma_{n}\right)} \\
& \leq \frac{L}{2}|y(t)-\tilde{y}(t)| e^{-h_{n}\left(t-\gamma_{n}\right)}+\frac{L}{2}|y(\pi(t))-\tilde{y}(\pi(t))| e^{-h_{n}\left(\pi(t)-\gamma_{n}\right)}+2 \tilde{\omega}_{1 n} \gamma_{n}|y-\tilde{y}|_{\gamma_{n}} \\
& +\widetilde{\omega}_{1 n} \int_{\gamma_{n}}^{t}(|y(s)-\tilde{y}(s)|+|y(\sigma(s))-\tilde{y}(\sigma(s))|) e^{-h_{n}\left(t-\gamma_{n}\right)} d s \\
& \leq L|y-\tilde{y}|_{h_{n}}+2 \tilde{\omega}_{1 n} \gamma_{n}|y-\tilde{y}|_{\gamma_{n}} \\
& +\widetilde{\omega}_{1 n} \int_{\gamma_{n}}^{t}\left(|y(s)-\tilde{y}(s)| e^{-h_{n}\left(s-\gamma_{n}\right)}+|y(\sigma(s))-\tilde{y}(\sigma(s))| e^{-h_{n}\left(\sigma(s)-\gamma_{n}\right)}\right) e^{h_{n}(s-t)} d s \\
& \leq L|y-\tilde{y}|_{h_{n}}+2 \tilde{\omega}_{1 n} \gamma_{n}|y-\tilde{y}|_{\gamma_{n}}+2 \widetilde{\omega}_{1 n}|y-\tilde{y}|_{h_{n}} \int_{\gamma_{n}}^{t} e^{h_{n}(s-t)} d s \\
& \leq L|y-\tilde{y}|_{h_{n}}+2 \tilde{\omega}_{1 n} \gamma_{n}|y-\tilde{y}|_{\gamma_{n}}+\frac{2 \tilde{\omega}_{1 n}}{h_{n}}|y-\tilde{y}|_{h_{n}}
\end{aligned}
$$


where $\widetilde{\omega}_{1 n}$ is as in the proof of Step 2, Theorem 3.1. We get

$$
|U y-U \tilde{y}|_{h_{n}} \leq\left(L+\frac{2 \widetilde{\omega}_{1 n}}{h_{n}}\right)|y-\tilde{y}|_{h_{n}}+2 \widetilde{\omega}_{1 n} \gamma_{n}|y-\tilde{y}|_{\gamma_{n}} .
$$

Combining (5.10)-(5.14), we deduce that

$$
\|U y-U \tilde{y}\|_{n} \leq\left(L+4 \gamma_{n} \widetilde{\omega}_{1 n}\right)|y-\tilde{y}|_{\gamma_{n}}+\left(L+\frac{2 \tilde{\omega}_{1 n}}{h_{n}}\right)|y-\tilde{y}|_{h_{n}} \leq \tilde{k}_{n}\|y-\tilde{y}\|_{n},
$$

where $\tilde{k}_{n}=\max \left\{L+4 \gamma_{n} \widetilde{\omega}_{1 n}, L+2 \widetilde{\omega}_{1 n} / h_{n}\right\}$. Choose

$$
0<\gamma_{n}<\min \left\{\frac{1-L}{4 \widetilde{\omega}_{1 n}}, n, \hat{\sigma}_{n}, \hat{\pi}_{n}\right\}, \quad h_{n}>\frac{2 \tilde{\omega}_{1 n}}{1-L},
$$

then we have $\tilde{k}_{n}<1$, by (5.15), $U$ is a $\tilde{k}_{n}$-contraction operator with respect to a family of seminorms $\|\cdot\|_{n}$.

(c) $C: X \rightarrow X$ is also completely continuous. We first show that $C$ is continuous. For any $y_{0} \in X$, let $\left(y_{m}\right)_{m}$ be a sequence in $X$ such that $\lim _{m \rightarrow \infty} y_{m}=y_{0}$.

Let $n \in \mathbb{N}^{*}$ be fixed. Put

$$
\begin{gathered}
K_{1}=\left\{\left(y_{m}+\xi\right)(s): s \in[0, n], m \in \mathbb{N}\right\}, \\
K_{2}=\left\{\left(y_{m}+\xi\right)(\chi(s)): s \in[0, n], m \in \mathbb{N}\right\} .
\end{gathered}
$$

Then $K_{1}, K_{2}$ are compact in $E$. For any $\epsilon>0$, since $G$ is continuous on the compact set $[0, n] \times[0, n] \times K_{1} \times K_{2}$, there exists $\delta>0$ such that for every $u_{i} \in K_{1}, v_{i} \in K_{2}, i=1,2$,

$$
\left|u_{i}-v_{i}\right|<\delta \Longrightarrow\left|G\left(t, s, u_{1}, v_{1}\right)-G\left(t, s, u_{2}, v_{2}\right)\right|<\frac{\epsilon}{n}, \quad \forall s, t \in[0, n] .
$$

Since $\lim _{m \rightarrow \infty} y_{m}=y_{0}$, there exists $m_{0}$ such that for $m>m_{0}$,

$$
\left|\left(y_{m}+\xi\right)(s)-\left(y_{0}+\xi\right)(s)\right|=\left|y_{m}(s)-y_{0}(s)\right|<\delta, \quad \forall s \in[0, n],
$$

and so

$$
\left|\left(y_{m}+\xi\right)(\chi(s))-\left(y_{0}+\xi\right)(\chi(s))\right|=\left|y_{m}(\chi(s))-y_{0}(\chi(s))\right|<\delta, \quad \forall s \in[0, n] .
$$

This implies that for all $t \in[0, n]$ and for all $m>m_{0}$,

$$
\begin{aligned}
& \left|C y_{m}(t)-C y_{0}(t)\right| \\
& \quad \leq \int_{0}^{t}\left|G\left(t, s,\left(y_{m}+\xi\right)(s),\left(y_{m}+\xi\right)(\chi(s))\right)-G\left(t, s,\left(y_{0}+\xi\right)(s),\left(y_{0}+\xi\right)(\chi(s))\right)\right| d s<\epsilon,
\end{aligned}
$$

so $\left|C y_{m}-C y_{0}\right|_{n}<\epsilon$, for all $m>m_{0}$, and the continuity of $C$ is proved. 
It remains to show that $C$ maps bounded sets into relatively compact sets. Now, let $\Omega$ be a bounded subset of $X$. We have to prove that for $n \in \mathbb{N}^{*},(C \Omega)_{n}$ is equicontinuous in $X_{n}$ and for every $t \in[0, n]$, the set $(C \Omega)_{n}(t)=\left\{\left.C y\right|_{[0, n]}(t): y \in \Omega\right\}$ is relatively compact in $E$.

Put

$$
\begin{gathered}
S_{1}=\{(y+\xi)(s): y \in \Omega, s \in[0, n]\}, \\
S_{2}=\{(y+\xi)(\chi(s)): y \in \Omega, s \in[0, n]\} .
\end{gathered}
$$

Then $S_{1}, S_{2}$ are bounded in $E$. Since $G$ is completely continuous, the set $G\left([0, n]^{2} \times S_{1} \times\right.$ $\left.S_{2}\right)$ is relatively compact in $E$, and so $G\left([0, n]^{2} \times S_{1} \times S_{2}\right)$ is bounded. Consequently, there exists $M_{n}>0$ such that

$$
|G(t, s,(y+\xi)(s),(y+\xi)(\chi(s)))| \leq M_{n}, \quad \forall t, s \in[0, n], \forall y \in \Omega .
$$

The rest of the proof runs as in (3.29), (3.31), and so $(C \Omega)_{n}=\left\{\left.C y\right|_{[0, n]}: y \in \Omega\right\}$ is equicontinuous and $(C \Omega)_{n}(t)$ is relatively compact in $E$ by

$$
\overline{(C \Omega)_{n}(t)} \subset t \overline{\mathrm{conv}} G\left([0, n]^{2} \times S_{1} \times S_{2}\right) .
$$

Using Lemma 3.3, $C(\Omega)$ is relatively compact in $X$. Therefore, $C$ is completely continuous.

(d) Finally, we also have that for all $n \in \mathbb{N} *$,

$$
\lim _{|y|_{n} \rightarrow \infty} \frac{|C y|_{n}}{|y|_{n}}=0
$$

For any given $\epsilon>0$, the assumptions $\left(\mathrm{I}_{3}\right),\left(\mathrm{I}_{4}\right)$ imply that there exists $\eta>0$ such that for all $t, s \in[0, n]$, for all $u, v \in E$, we get

$$
|G(t, s, u, v)| \leq \rho+\tilde{\omega}_{2 n}+\frac{\epsilon}{8 n}(|u|+|v|),
$$

where $\tilde{\omega}_{2 n}$ is also as in the proof of Step 2, Theorem 3.1. This implies that for all $t \in[0, n]$,

$$
\begin{aligned}
|C y(t)| & \leq \int_{0}^{t}|G(t, s,(y+\xi)(s),(y+\xi)(\chi(s)))| d s \\
& \leq n \rho+n \tilde{\omega}_{2 n}+\frac{\epsilon}{4}|\xi|_{n}+\frac{\epsilon}{4}|y|_{n} .
\end{aligned}
$$

It follows that if we choose $\mu_{n}>\max \left\{4 n \rho / \epsilon, 4 n \tilde{\omega}_{2 n} / \epsilon,|\xi|_{n}\right\}$, then for $|y|_{n}>\mu_{n}$, we have $|C y|_{n} /|y|_{n}<\epsilon$, this means that

$$
\lim _{|y|_{n} \rightarrow \infty} \frac{|C y|_{n}}{|y|_{n}}=0 .
$$

By applying Theorem 2.1, the operator $U+C$ has a fixed point $y$ in $X$. Then (5.1) has a solution $x=y+\xi$ on $(0, \infty)$. The result follows. 
Now, we also consider the asymptotically stable solutions for (5.1) defined as in Section 4. Here, we assume that $\left(\mathrm{I}_{1}\right)-\left(\mathrm{I}_{5}\right)$ hold and assume in addition that

(I $\left.\mathrm{I}_{6}\right) \pi(t)=t$, for all $t \in \mathbb{R}^{+}$;

( $\left.\mathrm{I}_{7}\right) V(t, s, 0,0)=0$, for all $(t, s) \in \Delta$;

$\left(\mathrm{I}_{8}\right)$ there exist two continuous functions $\omega_{3}, \omega_{4}: \Delta \rightarrow \mathbb{R}_{+}$such that

$$
|G(t, s, x, u)| \leq \omega_{3}(t, s)+\omega_{4}(t, s)(|x|+|u|), \quad \forall(t, s) \in \Delta, x, u \in E .
$$

Then, by Theorem $5.1,(5.1)$ has a solution on $[0, \infty)$.

On the other hand, if $x$ is a solution of (5.1), then $y=x-\xi$ satisfies (5.7). We note more that under the hypotheses $\left(\mathrm{I}_{1}\right),\left(\mathrm{I}_{6}\right)$, the function $\hat{f}$ turns out to be $f: \mathbb{R}_{+} \times E \rightarrow E$, satisfying $\left(\mathrm{A}_{1}\right)$. Consequently, for all $t \in \mathbb{R}_{+}$,

$$
\begin{aligned}
|y(t)| \leq & L|y(t)|+\int_{0}^{t} \omega_{1}(t, s)(|y(s)+\xi(s)|+|y(\sigma(s))+\xi(\sigma(s))|) d s \\
& +\int_{0}^{t}\left[\omega_{3}(t, s)+\omega_{4}(t, s)(|y(s)+\xi(s)|+|y(\chi(s))+\xi(\chi(s))|)\right] d s .
\end{aligned}
$$

It follows from (5.30) that for all $t \in \mathbb{R}_{+}$,

$$
\begin{aligned}
|y(t)| \leq & \frac{1}{1-L} \int_{0}^{t}\left(\omega_{1}(t, s)+\omega_{4}(t, s)\right)(|y(s)|+|y(\sigma(s))|+|y(\chi(s))|) d s \\
& +\frac{1}{1-L} \int_{0}^{t}\left(\omega_{1}(t, s)+\omega_{4}(t, s)\right)(|\xi(s)|+|\xi(\sigma(s))|+|\xi(\chi(s))|) d s \\
& +\frac{1}{1-L} \int_{0}^{t} \omega_{3}(t, s) d s,
\end{aligned}
$$

and so

$$
\begin{aligned}
|y(\sigma(t))| \leq & \frac{1}{1-L} \int_{0}^{\sigma(t)}\left(\omega_{1}(\sigma(t), s)+\omega_{4}(\sigma(t), s)\right)(|y(s)|+|y(\sigma(s))|+|y(\chi(s))|) d s \\
& +\frac{1}{1-L} \int_{0}^{\sigma(t)}\left(\omega_{1}(\sigma(t), s)+\omega_{4}(\sigma(t), s)\right)(|\xi(s)|+|\xi(\sigma(s))|+|\xi(\chi(s))|) d s \\
& +\frac{1}{1-L} \int_{0}^{\sigma(t)} \omega_{3}(\sigma(t), s) d s \\
\leq & \frac{1}{1-L} \int_{0}^{t}\left(\omega_{1}(\sigma(t), s)+\omega_{4}(\sigma(t), s)\right)(|y(s)|+|y(\sigma(s))|+|y(\chi(s))|) d s \\
& +\frac{1}{1-L} \int_{0}^{t}\left(\omega_{1}(\sigma(t), s)+\omega_{4}(\sigma(t), s)\right)(|\xi(s)|+|\xi(\sigma(s))|+|\xi(\chi(s))|) d s \\
& +\frac{1}{1-L} \int_{0}^{t} \omega_{3}(\sigma(t), s) d s,
\end{aligned}
$$

and it is similar to $|y(\chi(t))|$. 
Put $d(t)=|y(t)|+|y(\sigma(t))|+|y(\chi(t))|$. Then, combining these, for all $t \in \mathbb{R}_{+}$, we have

$$
d(t) \leq \int_{0}^{t} \theta(t, s) d(s) d s+e(t)
$$

where

$$
\theta(t, s)=\frac{1}{1-L}\left(\omega_{1}(t, s)+\omega_{4}(t, s)+\omega_{1}(\sigma(t), s)+\omega_{4}(\sigma(t), s)+\omega_{1}(\chi(t), s)+\omega_{4}(\chi(t), s)\right),
$$

$$
\begin{aligned}
e(t)= & \int_{0}^{t} \theta(t, s)[|\xi(s)|+|\xi(\sigma(s))|+|\xi(\chi(s))|] d s \\
& +\frac{1}{1-L} \int_{0}^{t}\left[\omega_{3}(t, s) d s+\omega_{3}(\sigma(t), s)+\omega_{3}(\chi(t), s)\right] d s .
\end{aligned}
$$

Using the inequality $(a+b)^{2} \leq 2\left(a^{2}+b^{2}\right)$, we get

$$
d^{2}(t) \leq 2 \int_{0}^{t} \theta^{2}(t, s) d s \int_{0}^{t} d^{2}(s) d s+2 e^{2}(t)
$$

Putting $z(t)=d^{2}(t), p(t)=2 \int_{0}^{t} \theta^{2}(t, s) d s,(5.36)$ is rewritten as follows:

$$
z(t) \leq p(t) \int_{0}^{t} z(s) d s+2 e^{2}(t)
$$

By (5.37), based on classical estimates, we also obtain

$$
d^{2}(t)=z(t) \leq 2 e^{2}(t)+p(t) e^{\int_{0}^{t} p(s) d s} \int_{0}^{t} 2 e^{-\int_{0}^{s} p(u) d u} e^{2}(s) d s, \quad \forall t \in \mathbb{R}_{+} .
$$

Then we have the following theorem about the asymptotically stable solutions.

Theorem 5.2. Let $\left(\mathrm{I}_{1}\right)-\left(\mathrm{I}_{8}\right)$ hold. Assume that

$$
\lim _{t \rightarrow \infty} 2 e^{2}(t)+p(t) e^{\int_{0}^{t} p(s) d s} \int_{0}^{t} 2 e^{-\int_{0}^{s} p(u) d u} e^{2}(s) d s=0,
$$

where

$$
\begin{aligned}
& p(t) \\
& \quad=\frac{2}{(1-L)^{2}} \int_{0}^{t}\left[\omega_{1}(t, s)+\omega_{4}(t, s)+\omega_{1}(\sigma(t), s)+\omega_{4}(\sigma(t), s)+\omega_{1}(\chi(t), s)+\omega_{4}(\chi(t), s)\right]^{2} d s,
\end{aligned}
$$

and $e(t)$ is defined as in (5.35).

Then every solution $x$ to (5.1) is an asymptotically stable solution. Furthermore,

$$
\lim _{t \rightarrow \infty}|x(t)-\xi(t)|=0 .
$$


Proof of Theorem 5.2. The proof is similar to that of Theorem 4.2. Let us omit here.

\section{Appendix}

Proof of Lemma 3.3. Assume that for each $n \in \mathbb{N}^{*}, A_{n}$ is equicontinuous in $X_{n}$ and for every $s \in[0, n]$, the set $A_{n}(s)=\left\{x(s): x \in A_{n}\right\}$ is relatively compact in $E$.

Let $\left(x_{k}\right)_{k}$ be a sequence in $A$. We will show that there exists a convergent subsequence of $\left(x_{k}\right)_{k}$.

In the Banach space $X_{n}=C([0, n], E)$, by $A_{n}$ being equicontinuous and for every $s \in$ $[0, n], A_{n}(s)=\left\{x(s): x \in A_{n}\right\}$ is relatively compact in $E$, so applying the Ascoli-Arzela theorem (see [5]), $A_{n}$ is relatively compact in $X_{n}$.

For $n=1$, since $\left(\mathrm{A}_{1}\right)$ is relatively compact in the Banach space $X_{1}=C([0,1], E)$, there exists a subsequence of $\left(x_{k}\right)_{k}$, denoted by $\left(x_{k}^{(1)}\right)_{k}$, such that

$$
\left(\left.x_{k}^{(1)}\right|_{[0,1]}\right)_{k} \longrightarrow x^{1} \quad \text { in } X_{1} \text {, as } k \longrightarrow \infty .
$$

For $n=2$, since $\left(\mathrm{A}_{2}\right)$ is relatively compact in the Banach space $X_{2}=C([0,2], E)$, there exists a subsequence of $\left(x_{k}^{(1)}\right)_{k}$, denoted by $\left(x_{k}^{(2)}\right)_{k}$, such that

$$
\left(\left.x_{k}^{(2)}\right|_{[0,2]}\right)_{k} \longrightarrow x^{2} \quad \text { in } X_{2} \text {, as } k \longrightarrow \infty \text {. }
$$

By the uniqueness of the limit, it is easy to see that $\left.x^{2}\right|_{[0,1]}=x^{1}$.

Thus, there exists a subsequence $\left(x_{k}^{(2)}\right)_{k}$ of $\left(x_{k}\right)_{k}$ such that

$$
\begin{gathered}
\left(\left.x_{k}^{(2)}\right|_{[0,1]}\right)_{k} \longrightarrow x^{1} \quad \text { in } X_{1} \text {, as } k \longrightarrow \infty, \\
\left(\left.x_{k}^{(2)}\right|_{[0,2]}\right)_{k} \longrightarrow x^{2} \quad \text { in } X_{2} \text {, as } k \longrightarrow \infty, \\
\left.x^{2}\right|_{[0,1]}=x^{1} .
\end{gathered}
$$

Therefore, for all $n \in \mathbb{N}^{*}$, by induction, we can establish a subsequence $\left(x_{k}^{(n+1)}\right)_{k}$ of $\left(x_{k}\right)_{k}$ such that

$$
\begin{gathered}
\left(\left.x_{k}^{(n+1)}\right|_{[0, m]}\right)_{k} \longrightarrow x^{m} \quad \text { in } X_{m}, \text { as } k \longrightarrow \infty, \forall m=\overline{1, n}, \\
\left(\left.x_{k}^{(n+1)}\right|_{[0, n+1]}\right)_{k} \longrightarrow x^{n+1} \quad \text { in } X_{n+1}, \text { as } k \longrightarrow \infty \\
\left.x^{n+1}\right|_{[0, m]}=x^{m}, \quad \forall m=\overline{1, n} .
\end{gathered}
$$

Put $y_{k}=x_{k}^{(k)}$. Then $\left(y_{k}\right)_{k}$ is a subsequence of $\left(x_{k}\right)_{k}$ and $\left(y_{k}\right)_{k}$ converges to $x$ in $X$, where $x$ is defined by

$$
x(t)=x^{n}(t) \quad \text { if } t \in[0, n], \forall n \in \mathbb{N}^{*} .
$$

The converse is obvious, and hence the lemma is proved. 


\section{Acknowledgments}

The authors wish to express their sincere thanks to Professor Klaus Schmitt and the referees for their helpful suggestions and comments.

\section{References}

[1] C. Avramescu, Some remarks on a fixed point theorem of Krasnosel'skii, Electronic Journal of Qualitative Theory of Differential Equations 2003 (2003), no. 5, 1-15.

[2] C. Avramescu and C. Vladimirescu, Asymptotic stability results for certain integral equations, Electronic Journal of Differential Equations 2005 (2005), no. 126, 1-10.

[3] T. A. Burton, A fixed-point theorem of Krasnosel'skii, Applied Mathematics Letters 11 (1998), no. $1,85-88$.

[4] T. A. Burton and C. Kirk, A fixed point theorem of Krasnosel'skii-Schaefer type, Mathematische Nachrichten 189 (1998), 23-31.

[5] J. Dieudonné, Foundations of Modern Analysis, Academic Press, New York, 1969.

[6] L. H. Hoa and K. Schmitt, Fixed point theorems of Krasnosel'skii type in locally convex spaces and applications to integral equations, Results in Mathematics 25 (1994), no. 3-4, 290-314.

[7] _. Periodic solutions of functional-differential equations of retarded and neutral types in Banach spaces, Boundary Value Problems for Functional-Differential Equations (J. Henderson, ed.), World Scientific, New Jersey, 1995, pp. 177-185.

[8] M. A. Krasnosel'skii, Topological Methods in The Theory of Nonlinear Integral Equations, Pergamon Press, New York, 1964.

[9] E. Zeidler, Nonlinear Functional Analysis and Its Applications. I, Springer, New York, 1986.

Le Thi Phuong Ngoc: Department of Natural Science, Nha Trang Educational College,

01 Nguyen Chanh Street, Nha Trang City, Vietnam

E-mail address: phuongngoccdsp@dng.vnn.vn

Nguyen Thanh Long: Department of Mathematics and Computer Science, University of Natural Science, Vietnam National University, 227 Nguyen Van Cu Street, Dist. 5, Ho Chi Minh, Vietnam

E-mail address: longnt@hcmc.netnam.vn 